TITLE:

\title{
Phase description of nonlinear dissipative waves under space-time-dependent external forcing
}

\author{
$\operatorname{AUTHOR}(S)$ : \\ Tonosaki, Y.; Ohta, T.; Zykov, V.
}

\section{CITATION:}

Tonosaki, Y. ... [et al]. Phase description of nonlinear dissipative waves under space-time-dependent external forcing Physica D: Nonlinear Phenomena 2010, 239(17): 1718-1722

ISSUE DATE:

2010-09-01

URL:

http://hdl.handle.net/2433/128924

\section{RIGHT:}

(c) 2010 Elsevier B.V.; この論文は出版社版でありません。引用の際には 出版社版をご確認ご利用ください。; This is not the published version. Please cite only the published version. 


\title{
Phase description of nonlinear dissipative waves under space-time dependent external forcing
}

\author{
Y. Tonosaki ${ }^{\mathrm{a}}$, T. Ohta ${ }^{\mathrm{a}, *}$, V. Zykov ${ }^{\mathrm{b}}$ \\ ${ }^{a}$ Department of Physics, Kyoto University, Kyoto, 606-8502 Japan \\ ${ }^{b}$ Institut fuer Theoretische Physik, Technische Universitaet Berlin, Hardenbergstrasse 36, D-10623 Berlin, Germany
}

\begin{abstract}
Based on the model system undergoing phase separation and chemical reactions, we investigate the dynamics of propagating dissipative waves under external forcing which is periodic both in space and time. A phase diagram for the entrained and non-entrained states under the external forcing is obtained numerically. Theoretical analysis in terms of phase description of the traveling waves is carried out to show that the transition between the entrained and the non-entrained states by changing the external frequency occurs either through a saddle-node bifurcation or through a Hopf bifurcation and that these two bifurcation lines are connected at a Bogdanov-Takens bifurcation point.
\end{abstract}

Keywords: Nonlinear dynamics, Pattern formation, Phase dynamics, Nonlinear dissipative waves, External forcing, Hopf bifurcation

\section{Introduction}

Synchronization and entrainment of nonlinear oscillators under external periodic forcing have been studied for many years. It has been shown that the phase dynamics which introduces one phase variable for a limit cycle oscillation is very useful to understand those phenomena $[1,2]$. The time-evolution equation for the phase $\theta$ is given by

$$
\frac{d \theta}{d t}=\Omega-\omega+f(\theta),
$$

where $\omega$ is the frequency of the limit cycle oscillation and $\Omega$ is that of the periodic external forcing. The function $f(\theta)$ is a $2 \pi$-periodic function. It is evident that Eq. (1) for $0<\theta<2 \pi$ has a pair of time-independent solutions for small differences of $|\omega-\Omega|$. One is stable and the other is unstable. If the value $|\omega-\Omega|$ is increased by changing the external frequency $\Omega$, the pair of solutions converges and disappears. This means that the bifurcation is a saddlenode bifurcation.

In comparison with these studies of nonlinear oscillators, nonlinear dissipative waves under external forcing have not been explored extensively despite the fact that existence of such waves are one of the most relevant self-organized phenomena far from equilibrium. Some recent studies towards this direction are given in refs. [3-8]. In the previous papers [9, 10], we addressed this problem not only for the external forcing but also for the feedback control. We carried out numerical simulations and theoretical analysis

\footnotetext{
*Corresponding author.Tel.:+81757533740; Fax:+81757533819

Email addresses: tonosaki@ton.scphys.kyoto-u.ac.jp (Y.

Tonosaki), takao@scphys.kyoto-u.ac.jp (T. Ohta)
}

based on a model system in one dimension. In the present paper, we focus our analysis on the external forcing and investigate the entrained dynamics in further detail.

In the next section (section 2) we start with description of our model system. Numerical results are shown in section 3. The phase dynamics approach is given in section 4. The section 5 is devoted to discussion.

\section{Model equations}

We start with the coupled set of equations for the local concentrations in a hypothetical ternary mixture where both phase separation and chemical reactions take place simultaneously. Let us assume that molecules A, B and $\mathrm{C}$ are adsorbed on a flat substrate with the local concentrations, $\psi_{A}, \psi_{B}$ and $\psi_{C}$, respectively. The other chemical species involved in the chemical reactions are assumed to exist abundantly in the gas phase above the substrate, and the products are also assumed to dissolve quickly into the gas phase. Each lattice site of the substrate is occupied by one and only one molecule A, B or C. Any pair of molecules $\mathrm{A}$ and $\mathrm{B}$ that are nearest neighbors exchange their positions randomly with a certain probability, but $\mathrm{C}$ molecules do not participate in such exchanges. In this way, the condition $\psi_{A}+\psi_{B}+\psi_{C}=1$ is satisfied in the continuum limit, while diffusion is exhibited by $\mathrm{A}$ and $\mathrm{B}$ molecules but not $\mathrm{C}$ molecules. When these molecules encounter other molecules in the gas phase, they undergo the chemical reactions $A \rightarrow B \rightarrow C \rightarrow A$ with the reaction rates $\gamma_{1}, \gamma_{2}$ and $\gamma_{3}$ respectively. It is assumed that the $\mathrm{A}$ and $\mathrm{B}$ species tend to segregate each other whereas the $\mathrm{C}$ component is neutral to both A and B. Then the time-evolution 
equations for the local concentrations $\psi=\psi_{A}-\psi_{B}$ and $\phi=\psi_{A}+\psi_{B}$ are given by [11].

$$
\begin{aligned}
\frac{\partial \psi}{\partial t} & =\nabla^{2}\left[-\nabla^{2} \psi-\tau \psi+\psi^{3}\right] \\
& +a_{1} \psi+a_{2} \phi+a_{3}+\Gamma(x, t), \\
\frac{\partial \phi}{\partial t}= & b_{1} \psi+b_{2} \phi+b_{3}+\Gamma(x, t) .
\end{aligned}
$$

The phase separation process is characterized by the parameter $\tau>0$. The coefficients are given in terms of the reaction rates by

$$
\begin{aligned}
& a_{1}=-\left(\gamma_{1}+\frac{\gamma_{2}}{2}\right), \\
& a_{2}=-\left(\gamma_{1}-\frac{\gamma_{2}}{2}+\gamma_{3}\right), \\
& a_{3}=b_{3}=\gamma_{3}, \\
& b_{1}=\frac{\gamma_{2}}{2} \\
& b_{2}=-\left(\frac{\gamma_{2}}{2}+\gamma_{3}\right) .
\end{aligned}
$$

The function $\Gamma(x, t)$ stands for the external force which is moving steadily to the right

$$
\Gamma(x, t)=\varepsilon \cos \left(q_{f} x-\Omega t\right),
$$

with the strength $\varepsilon$, the wave number $q_{f}$ and the frequency $\Omega$ [12]. Here we suppose that the system is exposed by illuminating light through a periodically arrayed slit and the slit moves at a constant velocity $\Omega / q_{f}$. As a result, we assume that the reaction rate $\gamma_{3}$ is modified such that $\gamma_{3} \rightarrow \gamma_{3}+\Gamma$. In this way, the $\Gamma$ term is added both in Eqs. (2) and (3) since $a_{3}=b_{3}=\gamma_{3}$ as Eq. (6). We have ignored a term $\Gamma \phi$ arising from the $\gamma_{3} \phi$ term in Eqs. (5) and (8) assuming a sufficiently small forcing $\epsilon$.

We have studied earlier the solution of Eqs. (2) and (3) without the external forcing [11]. The uniform timeindependent solution becomes unstable by increasing the parameter $\tau$ with fixing other parameters. Depending on the rate constants, e.g., $\gamma_{3}$, there are two possibilities. One is a Hopf bifurcation at a finite wave number. We have verified that a traveling wave appears above the threshold. The other is a Turing-type bifurcation beyond which a spatially periodic motionless pattern appears.

Throughout this paper, we will fix the parameters as $\tau=1.6, \gamma_{1}=0.3, \gamma_{2}=0.16$ and $\gamma_{3}=0.05$. This set of the parameters are close to the Hopf bifurcation threshold $\tau=\tau_{c}=1.46$ at a finite wave number $q=q_{c} \approx 0.9$ [11]. The frequency of oscillation at the bifurcation point is given by $\omega_{c} \approx 0.07$ and the external frequency $\Omega$ is varied around this critical frequency to investigate the dynamics under forcing.

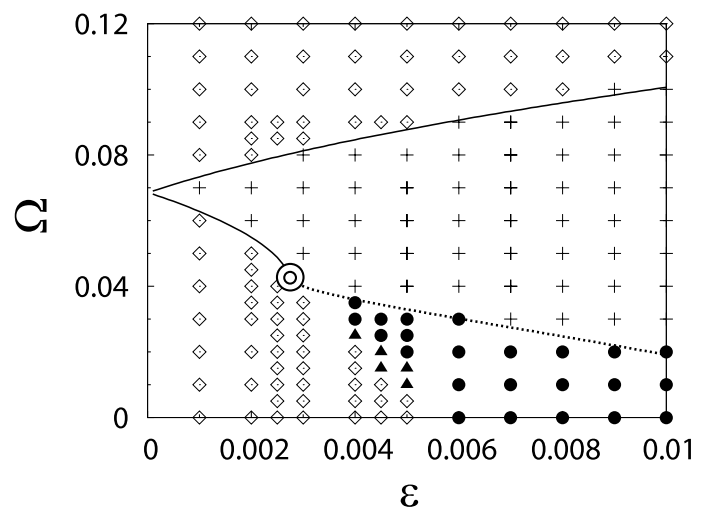

Figure 1: Phase diagram for the entrainment with the external forcing traveling to the right on the $\varepsilon-\Omega$ plane. The meaning of the symbols is given in the text. The solid lines are the saddle-node bifurcation threshold whereas the dotted line is the Hopf bifurcation threshold. These two lines are obtained from the phase equations of motion (12) and (13). The Bogdanov-Takens bifurcation point is indicated by the double circle.

\section{Numerical simulations}

We have carried out numerical simulations of Eqs. (2) and (3) with (9) in one dimension. The system size is $L=20 \pi$ with a periodic boundary condition and the space is divided into $N=128$ cells with the cell size $\delta x=20 \pi / N$. This system size is almost commensurate with the critical spatial period of the traveling wave $\ell_{c}=2 \pi / q_{c} \approx 2 \pi / 0.9$. The wave number of the external force is fixed to be the same as $q_{c}$ in order to avoid extra complications of dynamics. The explicit Euler scheme is employed with the time increment $\delta t=0.001$. Initially we provide a wave propagating to the right without the external forcing and then, at a certain time instant, switch on the external force (9) which is also traveling to the right.

Figure 1 represents the phase diagram on the $\varepsilon-\Omega$ plane obtained numerically asymptotically in time. The traveling wave is completely entrained by the external force in the region filled by symbols $(+)$ whereas it is not entrained in the region filled by $\diamond$. In the region indicated by $\bullet$, the wave trains undergo an oscillation trapped at the potential minima of the traveling external force. The space-time plot of these dynamics for $\varepsilon=0.007$ is displayed in Fig. 2 where the gray scale indicates the magnitude of $\psi$. The entrained state $(\Omega=0.07)$ is shown in Fig. 2(a). Fig. 2(b) illustrates the drift state $(\Omega=0.1)$ where the wave speed is modulated periodically every time the external force catches up the traveling waves. Fig. 2(c) exhibits the trapped state $(\Omega=0.02)$ where each wave train moves back and forth propagating gradually to the right on an average. In the narrow region indicated by the black triangles in Fig. 1, propagation reversal occurs. That is, the wave propagating to the right starts to propagate to the left after applying the external force which is propagating to the right. The mechanism of this apparently strange phenomenon will be clarified in section 4 . In particular, see Fig. 6 below. 
(a)

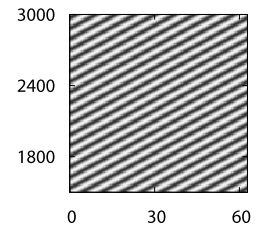

(b)

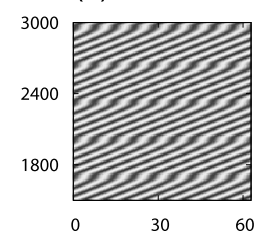

(c)

Figure 2: Space (horizontal)-time (vertical) plot of the propagating waves for $\varepsilon=0.007$ (a) in the entrained state for $\Omega=0.07$, (b) in the non-entrained state near the saddle-node bifurcation for $\Omega=0.1$ and (c) in the trapped state near the Hopf bifurcation for $\Omega=0.02$.

The above results are obtained in the situation that the external forcing is traveling to the same direction as the propagating wave. It should be noted, however, that Eqs. (2) and (3) without the external forcing have the waves traveling both to the right and to the left depending on the initial condition. Therefore, it is interesting to see what dynamics appears when the force moving to the opposite direction is applied. The phase diagram in such a case is obtained numerically as shown in Fig. 3 where the white circles indicates the region that the waves keep propagating to the initial direction with the periodic modulation by the external force traveling to the opposite direction. That is, the waves are not entrained. In the region indicated by other symbols, the waves change their propagating direction after switching on the external force and the asymptotic dynamics are the same as those in Fig. 1.

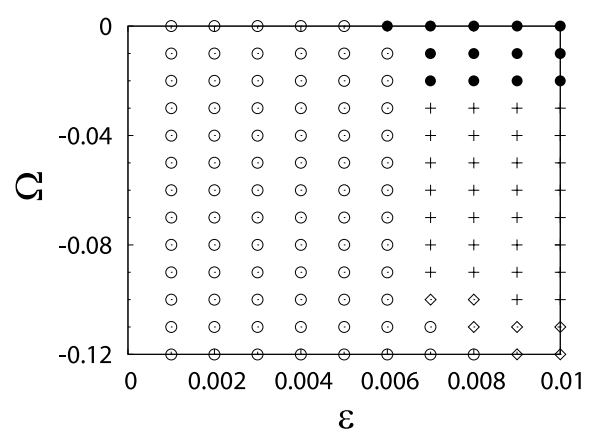

Figure 3: Phase diagram on the $\varepsilon-\Omega$ plane for the wave train propagation under external forcing traveling to the left.

\section{Phase equations}

In order to clarify the dynamics in the phase diagram displayed in Fig. 1, we derive the phase equations of motion for the propagating waves under the external force. We represent the solutions of Eqs. (2) and (3) as

$$
\begin{gathered}
\psi=\psi_{0}+\psi_{1}(t) \cos \left(q_{c} x-\Omega t+\theta_{1}(t)\right), \\
\phi=\phi_{0}+\phi_{1}(t) \cos \left(q_{c} x-\Omega t+\theta_{2}(t)\right),
\end{gathered}
$$

where $\psi_{0}$ and $\phi_{0}$ are the solutions of $a_{1} \psi+a_{2} \phi+a_{3}=$ 0 and $b_{1} \psi+b_{2} \phi+b_{3}=0$. The unknown functions $\psi_{1}(t), \phi_{1}(t), \theta_{1}(t)$ and $\theta_{2}(t)$ are to be determined. The procedure and the results are the same as the previous ones [9]. The final set of phase equations is given by

$$
\begin{aligned}
\frac{d \theta_{1}}{d t} & =\Omega-\frac{\varepsilon}{\psi_{1}^{(0)}} \sin \left(\theta_{1}\right) \\
& -a_{2} \frac{\phi_{1}^{(0)}}{\psi_{1}^{(0)}}\left(1+\frac{\phi_{1}^{(1)}}{\phi_{1}^{(0)}}-\frac{\psi_{1}^{(1)}}{\psi_{1}^{(0)}}\right) \sin \theta_{12}, \\
\frac{d \theta_{2}}{d t} & =\Omega-\frac{\varepsilon}{\phi_{1}^{(0)}} \sin \left(\theta_{2}\right) \\
& +b_{1} \frac{\psi_{1}^{(0)}}{\phi_{1}^{(0)}}\left(1+\frac{\psi_{1}^{(1)}}{\psi_{1}^{(0)}}-\frac{\phi_{1}^{(1)}}{\phi_{1}^{(0)}}\right) \sin \theta_{12},
\end{aligned}
$$

where

$$
\theta_{12}=\theta_{1}-\theta_{2}
$$

and

$$
\begin{aligned}
\frac{3}{4}\left(\psi_{1}^{(0)}\right)^{2}= & q_{c}^{2}+\tau-\tau_{c} \\
& +\frac{1}{q_{c}^{2}}\left(a_{1}-\frac{a_{2} b_{1}}{b_{2}}\left(\cos \theta_{12}\right)^{2}\right), \\
\phi_{1}^{(0)}= & \left(-\frac{b_{1}}{b_{2}} \cos \left(\theta_{12}\right)\right) \psi_{1}^{(0)}, \\
\psi_{1}^{(1)}= & \frac{2 \varepsilon}{3 q_{c}^{2}\left(\psi_{1}^{(0)}\right)^{2}} \\
\times & \left(\cos \left(\theta_{1}\right)-\frac{a_{2}}{b_{2}} \cos \left(\theta_{2}\right) \cos \theta_{12}\right), \\
\phi_{1}^{(1)}= & \frac{\phi_{1}^{(0)}}{\psi_{1}^{(0)}} \psi_{1}^{(1)}-\frac{\varepsilon}{b_{2}} \cos \left(\theta_{2}\right) .
\end{aligned}
$$

In Eq. (15), the positive solution for $\psi_{1}^{(0)}$ should be chosen for consistency with the simulations.

When the external force is absent, Eqs. (12) and (13) become a single equation for $\theta_{12}$, which takes the following form

$$
\frac{d \theta_{12}}{d t}=b_{2} \tan \left(\theta_{12}\right)+\frac{a_{2} b_{1}}{2 b_{2}} \sin \left(2 \theta_{12}\right) .
$$

Note that the right hand side is an odd function of $\theta_{12}$ so that Eq. (19) has two stable solutions which correspond to the waves propagating either to the left or to the right because the original set of Eqs. (2) and (3) are invariant under the transformation $x \leftrightarrow-x$.

Equations (12) and (13) are valid up to $O(\varepsilon)$. We have omitted the higher harmonics in the derivations of these equations. However, those effects are verified numerically to be negligible. Putting $d \theta_{1} / d t=d \theta_{2} / d t=0$ in Eqs. (12) and (13), the time-independent solution, which corresponds to the entrained state, has been obtained numerically by the Newton method and the stability of the stationary solutions is analyzed. 
(a)

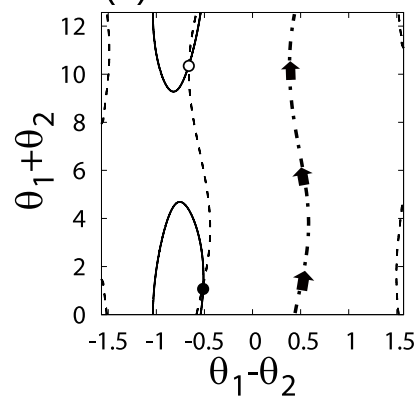

(b)

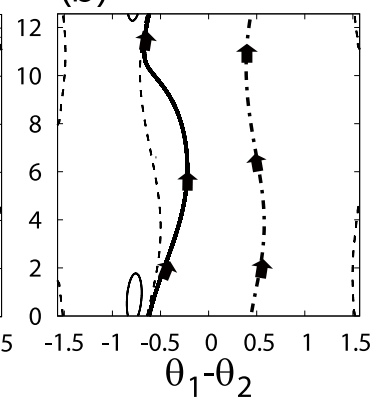

Figure 4: Nullcline of Eqs. (12) and (13) for $\varepsilon=0.004$ and (a) $\Omega=0.08$ and (b) $\Omega=0.09$ respectively. The white dot is the stable fixed point and the black dot is the unstable fixed point. This pair of the fixed points disappear in (b). The thin solid line is the line for $d \theta_{1} / d t=0$ and the thin broken line is the line for $d \theta_{2} / d t=0$. The thick solid line is the trajectory of the stable non-entrained solution propagating to the same direction as the external force. The dotted broken line is the trajectory of the stable non-entrained solution propagating to the opposite direction.

(a)

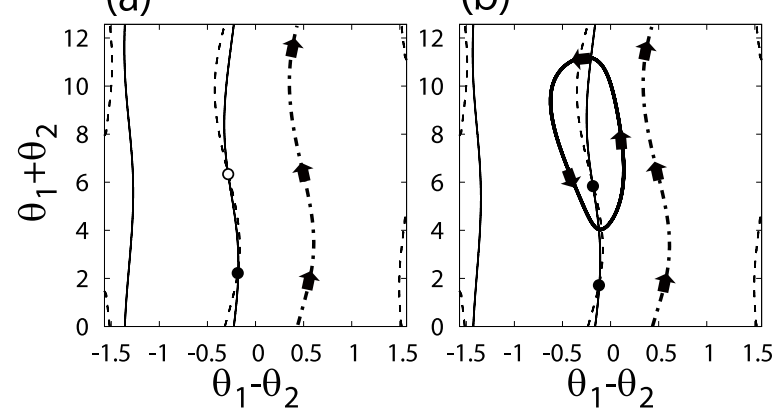

Figure 5: Nullcline and the asymptotic solutions of Eqs. (12) and (13) for $\varepsilon=0.004$ and (a) $\Omega=0.04$, and (b) $\Omega=0.029$ respectively. The thick solid line in (b) is the stable limit cycle solution. The meanings of other lines are the same as those in Fig. 4.

We have carefully examined the phase equations (12) and (13). It turns out that there are two different ways resulting in instability of the entrained state. One possibility is a pair annihilation of the stable and the unstable fixed points. The other one is that the eigen value in the linearized equations around the steady solution is complex and its real part becomes positive. In the previous paper [9], we overlooked this unusual possibility in the entrainment and synchronization phenomena. The former is a saddle-node bifurcation and the latter is a Hopf bifurcation. The solid lines in Fig. 1 indicates the saddle-node bifurcation line whereas the broken line is the Hopf bifurcation line. These two lines meet at about $\Omega=0.0430$ and $\varepsilon=0.00275$ which is a Bogdanov-Takens bifurcation point [13]. It is shown that these lines obtained from the phase (a)

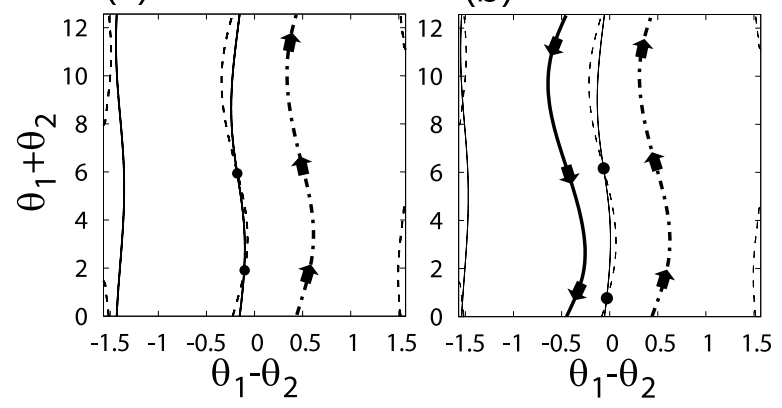

Figure 6: Nullcline and the asymptotic solutions of Eqs. (12) and (13) for $\varepsilon=0.004$ and (a) $\Omega=0.028$, and (b) $\Omega=0.01$ respectively. The meanings of the lines are the same as those in Fig. 4. Note that there is a dotted broken line but no thick solid line in (a) indicating that the non-entrained waves propagating to the same direction as the external force do not exist.

equations (12) and (13) are consistent quantitatively with the numerical simulations except for the larger values of $\epsilon(>0.09)$ where the lowest mode truncation in the phase dynamics becomes less accurate. In order to illustrate the above conclusion, the results of detailed computations of Eqs. (12) and (13) performed near the bifurcation lines are represented in Figs. 4, 5 and 6.

The nullclines of Eqs. (12) and (13) for $\varepsilon=0.004$ are plotted in the space of $\theta_{1}+\theta_{2}$ and $\theta_{12}=\theta_{1}-\theta_{2}$ in Figs. 4,5 and 6 for different values of the frequency $\Omega$. The thin solid (broken) line is the nullcline of Eq. (12) (Eq. (13)). Since Eqs. (12) and (13) are invariant under the simultaneous transformations $\theta_{12} \rightarrow \theta_{12}+\pi$ and $\theta_{1}+\theta_{2} \rightarrow \theta_{1}+\theta_{2}-\pi$, only the interval $-\pi / 2 \leq \theta_{12}<\pi / 2$ is plotted. Note that $\theta_{12}$ for $\theta_{1}+\theta_{2}=0$ is equal to $\theta_{12}$ for $\theta_{1}+\theta_{2}=4 \pi$ but $\theta_{1}+\theta_{2}$ for $\theta_{12}=-\pi / 2$ is equal to $\theta_{1}+\theta_{2}-\pi$ for $\theta_{12}=\pi / 2$.

Figures 4 (a) and (b) display the nullclines for $\Omega=0.08$ and for $\Omega=0.09$ respectively. There are a pair of steady solutions in Fig. 4 (a) where the solution indicated by the white dot is stable (node) and the one indicated by the black circle is unstable (saddle). These two solutions disappear for $\Omega=0.09$ in Fig. 4 (b) indicating a transition from the entrained state to the non-entrained state of the waves. It is evident that this transition occurs as a saddlenode bifurcation. The time-dependent trajectory corresponding to the non-entrained phase-slip waves is shown by the thick solid line in Fig. 4 (b). It should be noted that there is a stable non-entrained solution propagating to the direction opposite to the external forcing as the trajectory is indicated by the dotted broken line in Figs. 4 (a) and (b).

The arrows in Figs. 4, 5 and 6 indicate the direction of the time-evolution. The upward (downward) arrows show propagation to the left (right) relative to the external force propagating to the right. The arrows on the thick solid line in Fig. 4(b) are directed to upward because the wave is propagating to the right but its velocity is slower than that of the external force. 
The dynamics for the lower values of $\Omega$ are shown in Figs. 5 (a) and (b) for $\Omega=0.04, \Omega=0.029$ respectively. Figure 5 (a) for $\Omega=0.04$ displays a stable steady solution (white dot) of the entrained state and an unstable solution (black circle). For a smaller value of $\Omega=0.029$, both of the time-independent solutions are unstable (one is unstable focus and the other is saddle) and a limit cycle orbit appears around the first one that clearly indicates a Hopf bifurcation.

By decreasing further the value of $\Omega$, the limit cycle disappears and a trajectory of the monotonously timedependent motion appears which represents a periodically modulated traveling wave as shown by the dotted broken line for $\Omega=0.028$ in Fig. 6 (a) and both by the thick solid line and by the dotted broken line for $\Omega=0.01$ in Fig. 6 (b). Note that there is no thick solid line in Fig. 6(a) and that the non-entrained solution propagating to the same direction as the external force does not exist here. This means that the traveling wave changes the propagating direction when the external forcing (which is moving to the same direction as the initial traveling wave) is applied. Actually this phenomenon occurs for a narrow but finite interval of $\Omega$ as is indicated by the black triangles in Fig. 1.

(a)

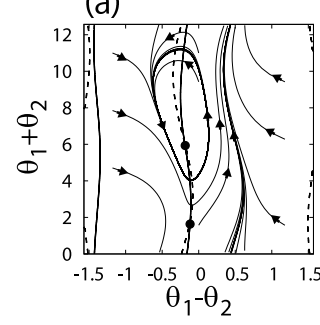

(b)

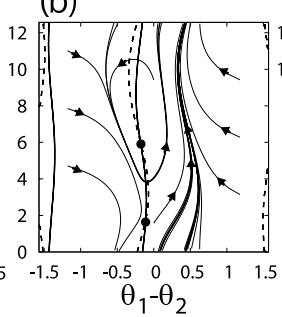

(c)

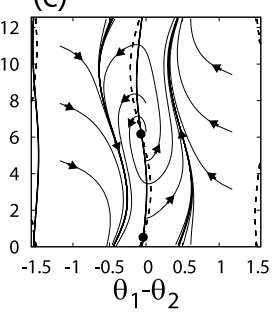

Figure 7: Trajectory on the $\theta_{1} \pm \theta_{2}$ plane corresponding to Eqs. (12) and (13) for $\varepsilon=0.004$ and (a) $\Omega=0.029$, (b) $\Omega=0.028$ and (c) $\Omega=0.01$.

In order to investigate the properties of the transitions from the trapped oscillation in Fig. 5 (b) to the reversal of propagation direction in Fig. 6 (a) and from the reversal of propagation to the phase-slip waves in Fig. 6 (b), we examine the phase trajectory on the $\theta_{1} \pm \theta_{2}$ plane as shown in Fig. 7 for $\epsilon=0.004$. It is evident that the limit cycle oscillation in Fig. 7 (a) for $\Omega=0.029$ disappears in Fig. 7 (b) for $\Omega=0.028$. This happens when the orbit touches, as $\Omega$ is decreased, the separatrix which exists between the limit cycle and the attractor of the nonentrained solution. One notes from Fig. 1 that decreasing $\Omega$ for $\Omega<\omega_{c}$ corresponds to decreasing the magnitude $\epsilon$ of the external force. Recall that the system is bistable for $\epsilon=0$ as mentioned in Eq. (19). This is the reason why two attractors of wave solutions propagating to the opposite direction appear for $\Omega=0.01$ together with the separatrix connecting two unstable fixed points as can be seen in Fig. 7 (c). The phase difference in the absence of the external force is $\theta_{12} \approx-0.628$ which is close to the location of the attractor of the waves propagating to the right (the trajectory directed downward). As a result, the right-moving wave emerges after switching on the external force.

\section{Discussion}

We have shown that the transition between entrained and non-entrained states occurs as a Hopf bifurcation as well as a saddle-node bifurcation. The latter is the usual behavior of the entrainment of a limit cycle oscillation under external periodic forcing. In the present paper, we have found, for the first time, that a Hopf bifurcation appears in the entrainment phenomenon of nonlinear dissipative waves. These results have been confirmed by the theory of phase description. The main results are summarized in the phase diagram shown in Fig. 1. It is mentioned here that the boundary between the drift state and the trapped oscillatory state is not so simple. For example, if one decreases the value of $\Omega$ for the fixed value of $\varepsilon=0.006$ the trapped oscillatory state disappears at $\Omega=0.013$ (although not shown in Fig. 1) but reappears for $\Omega \leq 0.002$.

It is emphasized that the two phase variables are necessary to represent the entrained and non-entrained dynamics properly. If only one phase variable is introduced for each oscillator as an ordinary phase dynamics of an oscillatory system $[1,2]$, the trapped oscillation as shown in Fig. 2(c) cannot be obtained theoretically. The above property inherent in the nonlinear propagating waves has a clear distinction from entrainment of limit cycle oscillation. When the external forcing is strong enough in the latter case, the original limit cycle orbit is deformed substantially so that the phase description becomes less accurate and the amplitude modulation have to be considered. Because of the coupling between the phase and the amplitude, an oscillatory modulation is possible [2]. However this oscillation has no relation to the trapped oscillation mentioned above caused by the coupling of the two phase variables.

The phase equations of motion given by Eqs. (12) and (13) are fairly complicated. One of the reasons is that the original system governed by Eqs. (2) and (3) have solutions propagating to the right and the left. When external forcing is absent, these two solutions are equally possible and therefore the system is bistable. The phase dynamics equations (12) and (13) contain this property correctly and therefore look complicated. As displayed in Fig. 6, Eqs. (12) and (13) exhibit the non-entrained waves propagating to the same direction as and the opposite direction to the external forcing.

In the present study, we have restricted ourselves to the case $q_{f}=q_{c}$ and varied the frequency of the external forcing $\Omega$ around the critical value $\omega_{c}$. In our previous paper [14], we have studied the dynamics for $q_{f} \neq q_{c}$. It has been shown that, if $q_{f} \approx q_{c}$ and $\Omega \approx \omega_{c}$, the traveling waves are 
entrained by the external force. However, if the deviations of $q_{f}$ and $\Omega$ are large, a wave-modulation occurs locally in space and it propagates either to the right or to the left depending on the parameters. This is a dynamical version of discommensuration in the commensurate- incommensurate transitions [15]. A trapped-oscillation of waves has also been obtained when the external frequency is substantially different from $\omega_{c}$ [14]. Therefore, it is interesting to investigate how the Bogdanov-Takens bifurcation point is extended when $q_{f} \neq q_{c}$. However, this is left for a future study.

\section{Acknowledgments}

This work was supported by the Grant-in-Aid for the priority area "Soft Matter Physics" from the Ministry of Education, Culture, Sports, Science and Technology (MEXT) of Japan and the Global COE Program "The Next Generation of Physics, Spun from Universality and Emergence". A part of this study was carried out at University of Bayreuth. The financial support from the Alexander von Humboldt foundation is gratefully acknowledged.

[1] Y. Kuramoto, Chemical Oscillations, Waves, and Turbulence, Springer, Berlin, Heidelberg, 1984.

[2] A. Pikovsky, M. Rosenblum, J. Kurths, Synchronization: A universal concept in nonlinear sciences, Cambridge University press, 2001.

[3] C. Utzny, W. Zimmermann, M. Bär, Europhys. Lett. 57 (2002) 113.

[4] S. Rüdiger, D.G. Miguez, A.P. Munuzuri, F. Sagués, J. Casademunt, Phys. Rev. Lett. 90, (2003) 128301.

[5] M. Henriot, J. Burguete, R. Ribotta, Phys. Rev. Lett. 91 (2003) 104501.

[6] D.G. Miguez, E.M. Nicola, A.P. Munuzuri, J. Casademunt, F. Sagués, L. Kramer, Phys. Rev. Lett. 93 (2004) 048303.

[7] F. M. Schneider, E. Schöll, M. A. Dahlem, Chaos 19 (2009) 015110 .

[8] M. Tlidi, A. G. Vladimirov, D. Pieroux, D. Turaev, Phys. Rev. Lett. 103 (2009) 103904.

[9] H. Tokuda, V. S. Zykov, T. Ohta, Phys. Rev. E 75 (2007) 066203.

[10] Y. Tonosaki, H. Tokuda, V. Zykov, T. Ohta, Europhys. Lett. $83(2008) 50011$.

[11] T. Okuzono, T. Ohta, Phys. Rev. E 67 (2003) 056211.

[12] T. Ohta, H. Tokuda, Phys. Rev. E 72 (2005) 046216.

[13] F. C. Hoppensteadt, E. M. Izhikevich, Weakly Connected Neural Networks, Springer, Berlin, 1997.

[14] H. Tokuda, T. Ohta, J. Phys. Soc. Jpn, 75 (2006) 064005.

[15] W. L. McMillan, Phys. Rev. B 14 (1976) 1496. 